\title{
LEACH Improved for Energy-Efficient Routing in Wireless Sensor Networks
}

\author{
Jignya Bhaumik Choksi ${ }^{1}$, Chirag Suryakant Thaker $^{2}$
}

\begin{abstract}
A new idea is proposed in this paper for improvement in the Low Energy Adaptive Clustering Hierarchy (LEACH) protocol. The cluster heads are selected based on the residual energy at each node at the end of each round. The expected number of cluster heads is kept stable considering the average residual energy of nodes which have not been cluster heads and the initial energy of a node. This work is carried out at each node level at the end of each round. Simulations are carried out considering the modified threshold function and the results are compared with the existing LEACH and family protocols.
\end{abstract}

Keywords: Data fusion, $L E A C H$, initial energy, residual energy

\section{Introduction}

Wireless Sensor Networks generally consist of one or more sinks (or base stations) and perhaps tens or thousands of sensor nodes scattered in a physical space [1]. The sensor node measures environmental conditions and transforms them into electrical signals. This information is then sent to a sink through radio transceiver. Sensor networks are distributed sensors to monitor conditions like temperature, sound, vibration, pressure and pollutants etc [2]. The sink in turn queries the sensor nodes for information. WSNs are mostly used in, low bandwidth and delay tolerant applications ranging from civil and military to environmental and healthcare monitoring. WSNs form a link between the physical world and digital data network. They provide a distributed network. In WSNs, the node resource is highly constrained [3]. Data aggregation can be used for reducing energy consumption [4] [5]. Data aggregation, also known as data fusion, can combine multiple data packets received from different sensor nodes. It reduces the size of the data packet by eliminating the redundancy. Wireless communication cost is also decreased by the reduction in the data packets [6]. Routing protocols play an important role to increase the performance of wireless sensor networks. Several routing protocols have been designed and implemented to achieve high lifetime of networks and reduce the energy consumption. Low Energy Adaptive Clustering Hierarchy (LEACH) [7] is the first implemented cluster-based routing protocol and it is also considered as the base of other cluster routing protocols. Some other important protocols are PEGASIS [8], TEEN [9], APTEEN[10], HEED[11], LEACH-C [12], ALEACH [13].

1. Leach

\section{Leach And Family Protocols}

LEACH (Low-Energy Adaptive Clustering Hierarchy) is a clustering-based protocol that utilizes randomized rotation of local cluster base stations (cluster-heads) to evenly distribute the energy load among the sensors in the network [7]. Working of LEACH protocols is carried out in the form of rounds. Each round consists of a set-up phase (in which the clusters are formed) and a steady-state phase (in which data transmission takes place). Clusters are formed in LEACH by a distributed algorithm. The decisions made by nodes are not dependent on any centralized control [12]. A node makes this decision based on the expected percentage of cluster-heads in the network, which is determined beforehand, and the number of times the node has become a cluster-head till that time. Each node generates a random number between 0 and 1 . If the number generated is less than a threshold value $\mathrm{P}_{i}(\mathrm{t})$, the node is chosen to become a cluster-head for the current round. Each node should choose to become a cluster head at round $r$ with probability

$\mathrm{P}_{i}(\mathrm{t})= \begin{cases}\frac{k}{N-k *\left(r \bmod \frac{N}{k}\right)} & C_{i}(t)=1 \\ 0 & C_{i}(t)=0\end{cases}$

where $\mathrm{k}$ is the expected number of cluster-heads, $\mathrm{N}$ is the number of nodes in the network, $\mathrm{r}$ is the current round. $\mathrm{C}_{\mathrm{i}}(\mathrm{t})$ is 0 if the node $\mathrm{i}$ has already been a cluster-head and 1 otherwise. All the nodes will have been cluster-heads after $\mathrm{N} / \mathrm{k}$ rounds and $\mathrm{C}_{\mathrm{i}}(\mathrm{t})$ will be set to 1 at this time. After this the next cycle begins. 


\section{LEACH-Centralized (LEACH-C)}

LEACH protocol does not guarantee about the number or cluster-head nodes or placement of nodes. Cluster-head nodes can be spread evenly in the network and better clusters can be formed by using a central control algorithm. LEACH-Centralized (LEACH-C) is described in [12]. LEACH-C uses a centralized clustering algorithm. The steady-state is same as that in LEACH. Each node sends information related to its current location to the base station. Also, information about the energy level of the node is sent along with it. The BS has the responsibility to decide the cluster-heads and form better clusters. It also takes care that the energy load is properly distributed among the nodes in the network. The average energy of the nodes is found by the sink and the nodes having the energy-level less than the average are not chosen to be the cluster-heads in the current round. The base station broadcasts the information related to the cluster-head ID of each node after the formation of clusters and finding the cluster-heads. If the cluster-head ID of a node matches its own ID, the node is a cluster-head. If it is not so, the node finds its slot of TDMA. The node will go to sleep when it is not having turn to transmit, otherwise it will send data.

\section{Advanced LEACH (ALEACH)}

ALEACH [13] introduces the consideration of current state energy of a node while making a decision whether a node can become a cluster-head or not. It modifies the equation of threshold of LEACH by introducing Current State probability $\left(\mathrm{CS}_{\mathrm{p}}\right)$. The threshold equation of a node for the current round depends on both terms. The current state probability of a node is set as a function of the current residual energy of a node $\left(\mathrm{E}_{\text {current }}\right)$ relative to the initial value of energy $\left(\mathrm{E}_{\mathrm{n}-\max }\right)$, multiplied by the percentage of clusters in the network $(\mathrm{k} / \mathrm{N})$. According to equation 1 , the threshold value is represented as

$$
\mathrm{T}(\mathrm{n})=\frac{k}{N-k *\left(r \bmod \frac{N}{k}\right)}+\frac{E_{\text {current }}}{E_{n-\max }} \times \frac{k}{N}
$$

where $\mathrm{N}$ is the number of nodes in the network, $\mathrm{k}$ is the expected number of cluster-heads and $\mathrm{r}$ is the current round number. In order to distribute energy dissipation evenly in the network, ALEACH selects a node with more amount of residual energy as a cluster-head over a node with less amount of residual energy.

\section{Analysis of LEACH, LEACH-C and ALEACH protocols}

The existing LEACH (Low Energy Adaptive Clustering Hierarchy) protocol is a good approach for energy-efficient routing mechanism for Wireless Sensor Networks. But it still has some drawbacks. Equation 1 depicts that all the nodes have the same probability of being elected as cluster-heads, irrespective of the amount of remaining energy they possess. It treats nodes with less residual energy in the same way as nodes with more amount of residual energy. As a result, in some cases, the nodes with low residual energy may be selected as cluster-head instead of nodes with high residual energy. This can result into improper distribution of network energy and the lifetime of the network might decrease. The existing LEACH protocol considers just static values for finding the threshold in order to decide whether a node can be elected as a cluster-head or not. It does not take into consideration the residual energy of the nodes while selecting the cluster-heads. If a node chosen to be cluster-head has less amount of residual energy, then it will die soon and such situations can lead to imbalance of energy in the network. Hence, it is essential to consider the residual energy of the nodes while making a decision to become a cluster-head. This can lead to proper balance of energy-levels of the nodes in the network, which can increase the lifetime of the network. It is not necessary that LEACH-C is better than LEACH. It might be more expensive than LEACH because of the central control by the base station. Each node needs to send its information to the base station. Such tasks in this protocol demand more energy. Hence, the performance of the network might reduce. Equation 2 in ALEACH generates a very large threshold. The number of cluster-heads might reduce considerably after some time. LEACH protocol ensures the optimal number of cluster-heads for each round by the generated threshold value. But the threshold generated by ALEACH does not ensure this. In this way, it cannot be said that the expected number of clusterheads will be maintained in ALEACH.

\section{The Proposed Improved LEACH protocol}

\section{Leach Improved Protocol}

The proposed algorithm is an improvement in LEACH. The residual energy of a node is considered while finding the threshold value. Hence, more the residual energy a node has, higher the probability it will be elected as a cluster-head. The threshold function of LEACH can be given as shown below: 
$\mathrm{P}_{i}(\mathrm{t})= \begin{cases}\frac{k}{N-k *\left(r \bmod \frac{N}{k}\right)} \frac{E_{\text {current }}}{E_{n-\max }} & C_{i}(t)=1 \\ 0 & C_{i}(t)=0\end{cases}$

where $E_{\text {current }}$ is the current residual energy of node $i, E_{n-m a x}$ equals to initial energy value, $k$ is the number of cluster-heads, $\mathrm{N}$ is the number of nodes in the network, $\mathrm{r}$ is the current round number, $\mathrm{C}_{\mathrm{i}}(\mathrm{t})=0$ if the node $\mathrm{i}$ has already been a cluster-head in the current cycle, otherwise $C_{i}(t)=1$. Above equation (3) may cause reduction in the number of cluster-heads when the energy level of the entire network is low. Hence, it is necessary to adjust the expected number of cluster-heads back to $\mathrm{k}$ as mentioned in LEACH. In order to do so, the improved algorithm sets the interval of random number generation as $\left[0, E_{\text {average }} / E_{n-m a x}\right][14] . E_{\text {average }}$ is the average of residual energy levels found at the end of previous round considering the nodes that have not already been cluster-heads in the current cycle. In [14] work is carried out at base-station. Instead, the proposed protocol carries out the work at each node level at the end of each round.

2. Design and Working of Improved LEACH protocol

The design of the improved LEACH protocol can be explained by the flowchart shown in Fig. 1. The proposed improved LEACH protocol forms clusters by using a distributed algorithm. The operation of the algorithm is divided into rounds. Each round begins with a set-up phase when the clusters are organized, followed by a steady-state phase when data are transferred from the nodes to the BS via their respective clusterheads. The nodes organize themselves into local cluster, with one node acting as the cluster-head. All noncluster-head nodes transmit their data to the cluster-head, while the cluster-head node receives data from all the cluster members, performs signal processing functions for data aggregation on the data and transmit data to the remote BS. Being a cluster-head node is much more energy intensive than being a non cluster-head node. Hence, if cluster-heads are selected in a better way, it can result into proper balance of energy in the network and increase the lifetime and performance of the network. The improved protocol considers the residual energy of a node while making a decision to select it as a cluster-head or not. The threshold calculated depends on the current residual energy of a node. Thus, it helps to make appropriate decision as to select nodes with more residual energy as cluster-heads as compared to the nodes with less residual energy. This leads to even distribution of energy amongst the nodes. This is an important advantage of the improved protocol. The interval for random number generation is also modified so as to get expected number of cluster-heads as $\mathrm{k}$. Thus, the problem of continuous decrease in the number of cluster-heads which was found earlier with other protocols can be eliminated. Another advantage of the improved protocol is that the nodes make autonomous decisions whether to become a cluster-head or not, without any centralized control. Hence, no long-distance communication with the base station is required for making this decision. 


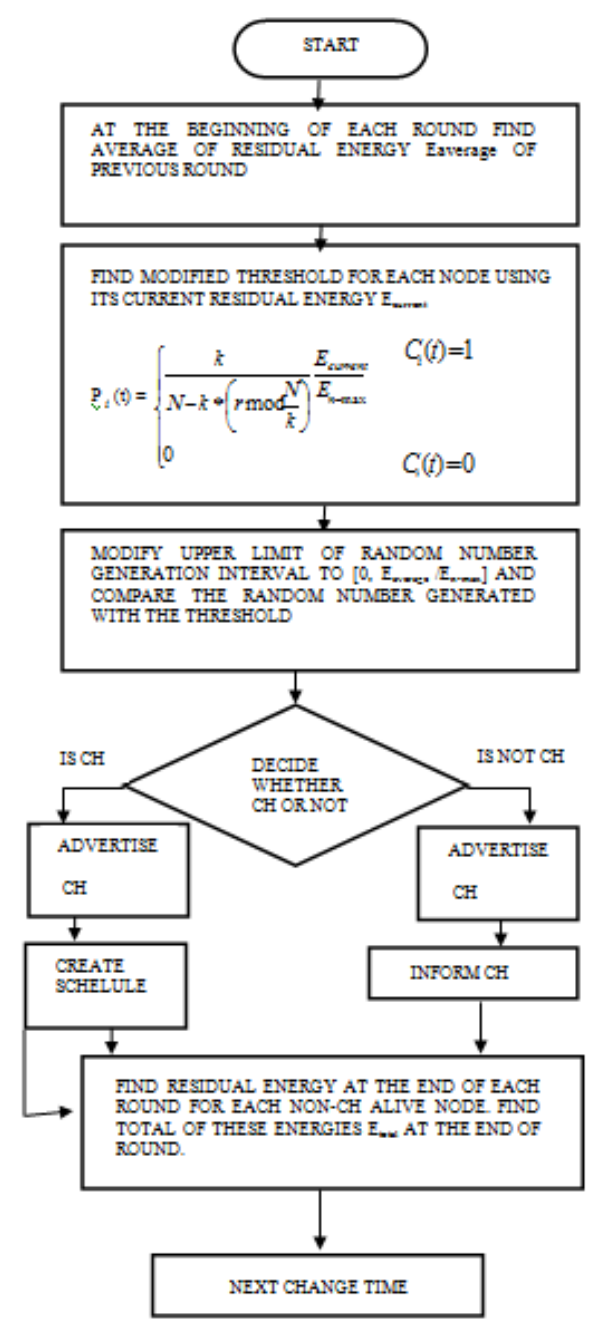

Figure 1 Working of IMPROVED LEACH

The modified protocol finds the residual energy of each node at the end of each round. Total of these residual energies $E_{\text {total }}$ is found at the end of the round. The nodes that are alive and have not been cluster-heads in the current cycle are only taken into consideration during this calculation. The threshold value generated takes into consideration residual energy for each node along with the initial energy value. At the beginning of each round, the average $\mathrm{E}_{\text {average }}$ of the residual energies of previous round is found using the $\mathrm{E}_{\text {total }}$ value. This average value is used to set the interval for random number generation in order to compare it with the threshold. The Setup and Steady phase of the improved protocol are as described below:

The Set-up phase: During the cluster Set-up phase, the improved protocol modifies the threshold function introduced in LEACH by introducing residual energy of a node. The modified algorithm also finds the average residual energy of nodes that have not been cluster-heads in the current cycle to modify the random number generation interval introduced in LEACH. The residual energy of the nodes that have not been clusterheads in current cycle is found at the end of steady phase each round. Total of these residual energies $\mathrm{E}_{\text {total }}$ is calculated at the end of the round. In the beginning of the set-up phase of the next round, the average residual energy $E_{\text {average }}$ of the previous round is calculated using this information and this average is utilized for finding upper-limit of the interval for random number generation. The random number generated will be compared with the threshold value for each node and based on this it will be decided whether the node can become cluster-head or not.

The Steady phase:The working of Steady phase of the improved algorithm is similar to that of LEACH. The steady-state operation is broken into frames in set-up phase, each frame is divided into many slots according to the number of the nodes in this cluster, and each node sends packet to its cluster-head in the slot allocated to it. Each non-cluster-head node uses power control to set the amount of transmits power based on the received strength of the cluster-head advertisement for reducing energy dissipation. At the end of each round after the Steady phase, residual energy of each alive non-cluster-head node is found and total of these energies 
$\mathrm{E}_{\text {total }}$ is calculated. This will be used in the Set-up phase of the next round to find the average residual energy $\mathrm{E}_{\text {average. }}$.

\section{Simulation Results And Analysis}

1. Simulation Environment: The performance of modified LEACH protocol is compared with LEACH, LEACH-C and ALEACH protocols in detail considering parameters like energy efficiency and lifetime of network. The simulation is carried out in NS2.34.

2. Simulation metrics and Results: To compare the performance of the modified LEACH algorithm with the original LEACH, LEACH-C and ALEACH, the following metrics are measured:

Energy consumption: An important factor for network load balancing is energy consumption. Uniform energy consumption can lead to less possibility for premature death of nodes. Also, less energy consumption per round will lead to better network performance.

Number of alive nodes: The number of alive nodes with respect to time in a network can determine network lifetime. If the lifetime of the network is more, then the network performs well and it can monitor the surrounding environment longer.

The modified protocol was simulated considering the simulation conditions as mentioned in Table 1.

Table 1 Simulation Parameters

\begin{tabular}{|l|c|}
\hline \multicolumn{1}{|c|}{ PARAMETER NAME } & PARAMETER VALUE \\
\hline Number of nodes (excluding the base station) & 100 \\
\hline Simulation Area (x,y) & 1000 X $1000 \mathrm{~m}^{2}$ \\
\hline Initial energy of each node & $5 \mathrm{~J}$ \\
\hline Relative position of the base station & $(50,175)$ \\
\hline The percentage of cluster-heads & $5 \%$ \\
\hline Simulation end condition & Number of nodes $<$ \\
\hline Round time & $20 \mathrm{sec}$ \\
\hline Simulation Time & $3600 \mathrm{sec}$ \\
\hline
\end{tabular}

The improved algorithm is simulated considering hundred nodes randomly deployed between $(\mathrm{x}=0$, $y=0)$ and $(x=1000, y=1000)$. The base station is located at $(50,175)$. The initial energy of each node is considered to be 5 Joule. The expected number of cluster heads in every round is 5 . The nextchangetime for each round is $20 \mathrm{sec}$.

Fig. 2 shown below is a graph of number of alive nodes of LEACH, LEACH-C, ALEACH and LEACH IMPROVED with elapse of time. It shows that as compared to LEACH, LEACH-C and ALEACH, in the LEACH IMPROVED algorithm the number of alive nodes is more with respect to time. The nodes die at a slower rate in the improved protocol. This is due to balanced energy distribution of nodes while deciding cluster-heads in the improved protocol. The consideration of residual energy in selection of cluster-heads makes it possible to make nodes with more residual energy as cluster-heads rather than nodes with less residual energy. The sudden decrease in energy of some nodes which was a drawback in earlier protocols will be avoided. This will eventually lead to increased life of the network.

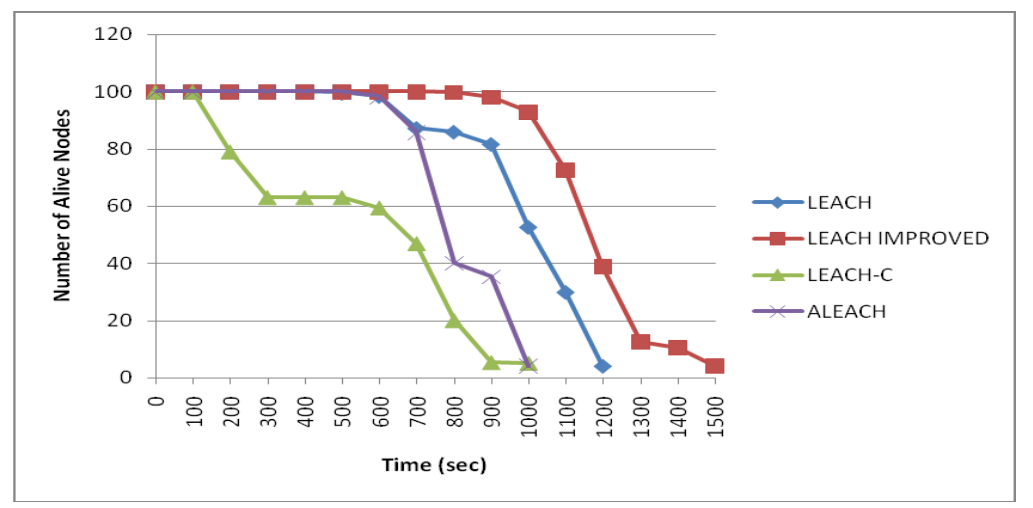

Figure 2 Graph of number of alive nodes over time

Fig. 3 shown below is a graph of energy consumption of LEACH, LEACH-C, ALEACH and LEACH IMPROVED with elapse of time. It shows that as compared to the other algorithms, the LEACH IMPROVED algorithm consumes less energy with respect to time. This is due to balanced energy distribution of nodes while deciding cluster-heads in the improved protocol. 


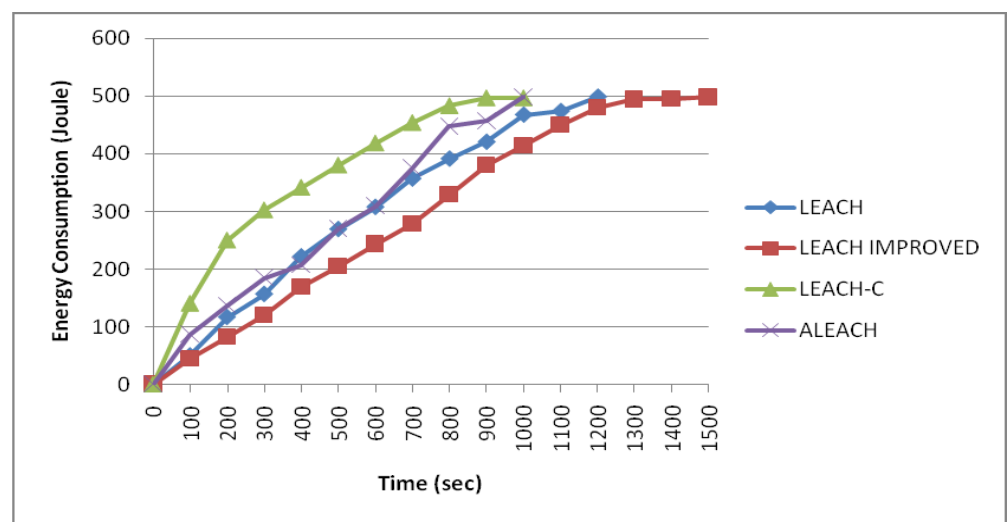

Figure 3 Graph of energy consumption over time

Simulations were carried out for LEACH, LEACH-C, ALEACH and LEACH IMPROVED varying the expected number of cluster-heads. The metrics measured were as mentioned below:

Lifetime of network: The lifetime of network can be measured in terms of number of alive nodes with respect to time in a network. In order to analyze the lifetime of network, the metrics considered are First Node Death (FND) time, Half Node Death (HND) time and Last Node Death (LND) time.

The simulation conditions are as mentioned in Table 2.

Table 2 Simulation Parameters

\begin{tabular}{|c|c|}
\hline PARAMETER NAME & PARAMETER VALUE \\
\hline Number of nodes (excluding the base station) & 100 \\
\hline Simulation Area $(\mathrm{x}, \mathrm{y})$ & $1000 \times 1000 \mathrm{~m}^{2}$ \\
\hline Initial energy of each node & $2 \mathrm{~J}$ \\
\hline Relative position of the base station & $(50,175)$ \\
\hline Round time & $20 \mathrm{sec}$ \\
\hline Simulation Time & $3600 \mathrm{sec}$ \\
\hline
\end{tabular}

Fig.4 shows graph of First Node Death time of a network for LEACH, LEACH-C, ALEACH and LEACH IMPROVED considering different number of cluster-heads.

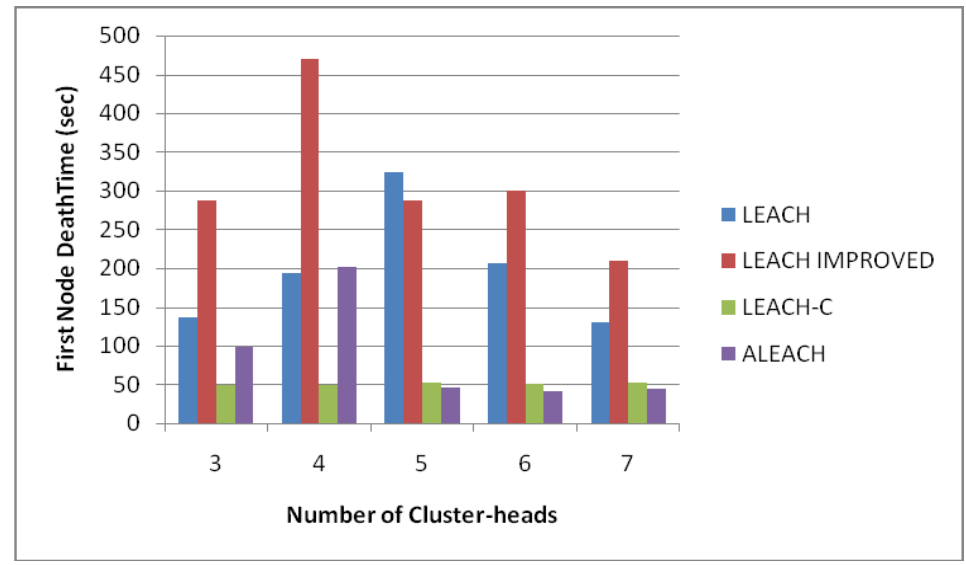

Figure 4 Graph of First Node Death time for varied number of cluster-heads

Fig. 5 shows graph of Half Node Death time of a network for LEACH, LEACH-C, ALEACH and LEACH IMPROVED considering different number of cluster-heads. 


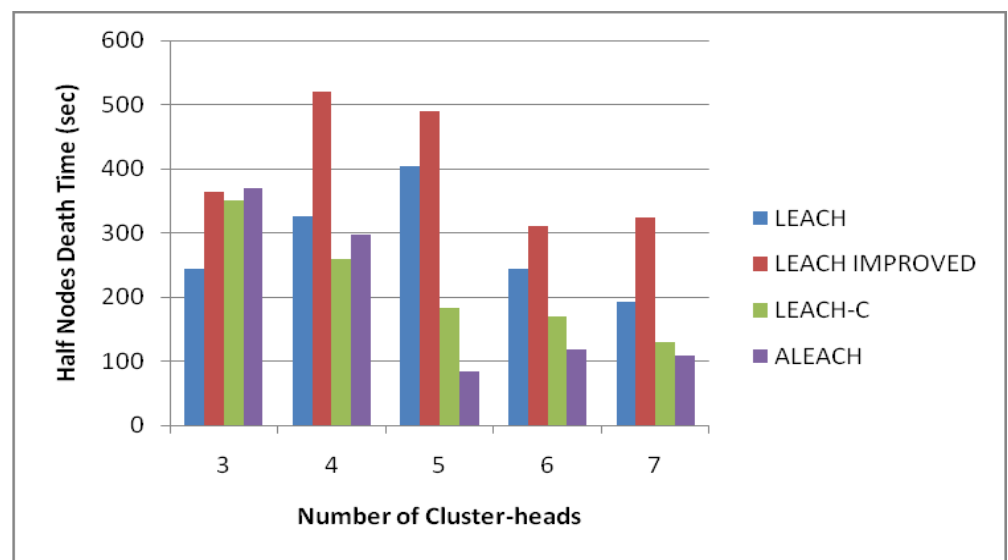

Figure 5 Graph of Half Node Death time for varied number of cluster-heads

Fig.6 shows graph of Last Node Death time of a network for LEACH, LEACH-C, ALEACH and LEACH IMPROVED considering different number of cluster-heads.

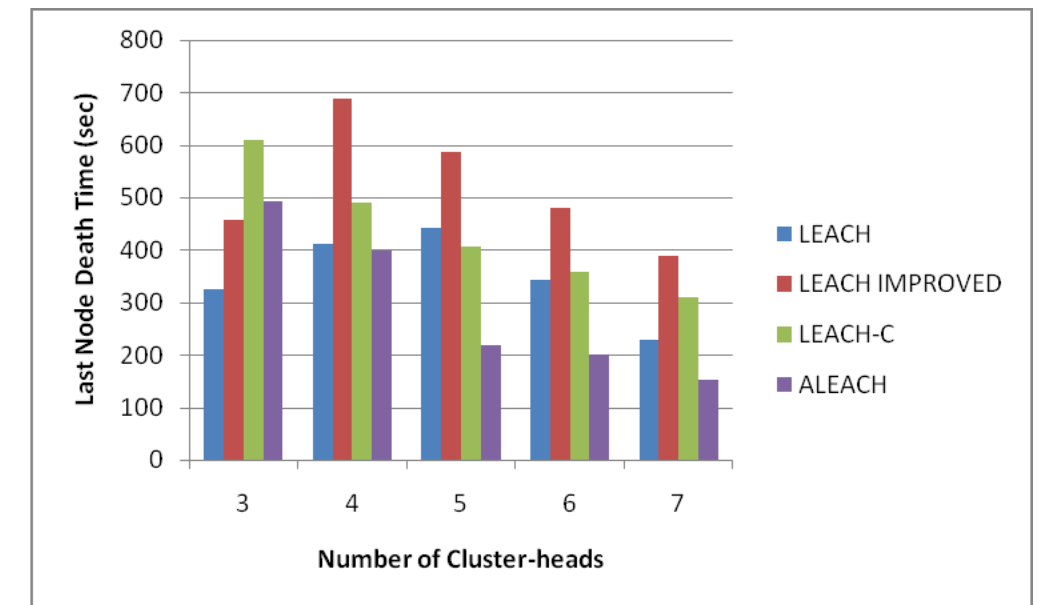

Figure 6 Graph of Last Node Death time for varied number of cluster-heads

From the above graphs it can be inferred that the expected number of cluster-heads when set as 4 , it gives best results for the improved protocol.

Similar simulations were carried out for LEACH, LEACH-C, ALEACH and LEACH IMPROVED varying the value of initial energy. The simulation conditions are as mentioned below in Table 3 .

Table 3 Simulation Parameters

\begin{tabular}{|l|c|}
\hline \multicolumn{1}{|c|}{ PARAMETER NAME } & $\begin{array}{c}\text { PARAMETER } \\
\text { VALUE }\end{array}$ \\
\hline Number of nodes (excluding the base station) & 100 \\
\hline Simulation Area $(\mathrm{x}, \mathrm{y})$ & $1000 \mathrm{X} 1000 \mathrm{~m}^{2}$ \\
\hline The percentage of cluster-heads & $5 \%$ \\
\hline Relative position of the base station & $(50,175)$ \\
\hline Round time & $20 \mathrm{sec}$ \\
\hline Simulation Time & $3600 \mathrm{sec}$ \\
\hline
\end{tabular}

Fig. 7 shows graph of First Node Death time of a network for LEACH, LEACH-C, ALEACH and LEACH IMPROVED considering different amount of initial energy. 


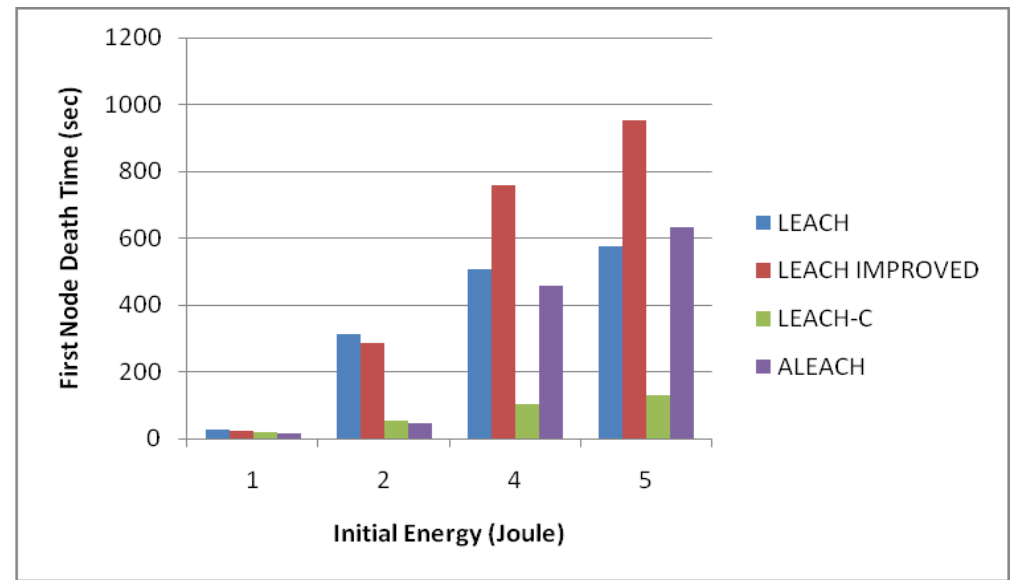

Figure 7 Graph of First Node Death time for different initial energy values

Fig. 8 shows graph of Half Node Death time of a network for LEACH, LEACH-C, ALEACH and LEACH IMPROVED considering different amount of initial energy.

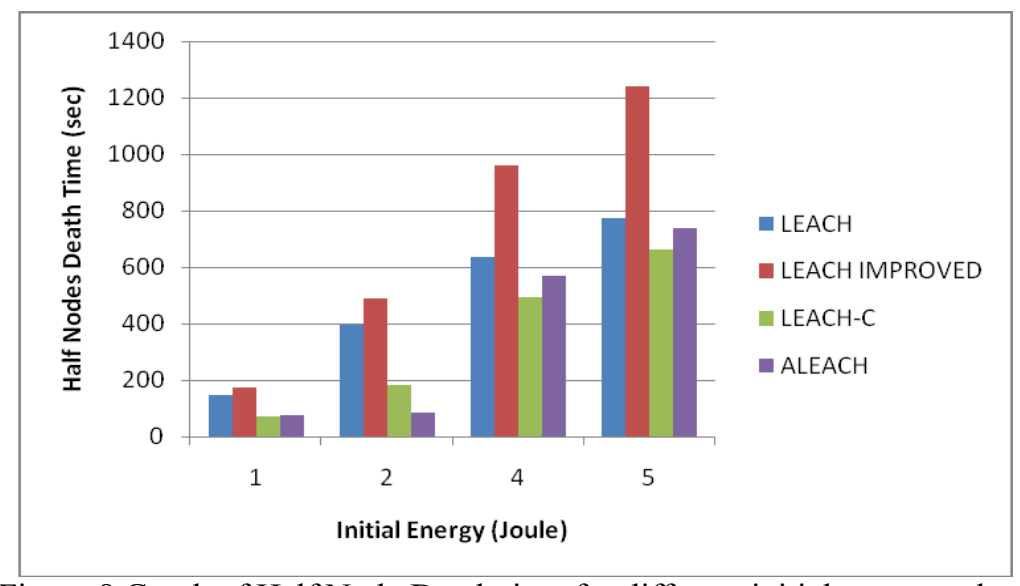

Figure 8 Graph of Half Node Death time for different initial energy values

Fig. 9 shows graph of Last Node Death time of a network for these protocols considering different amount of initial energy.

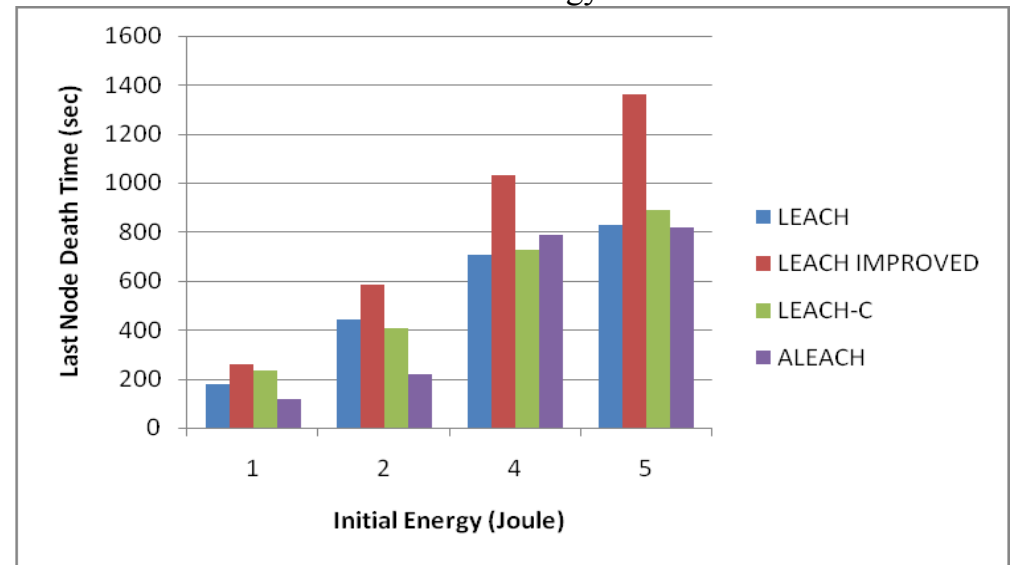

Figure 9 Graph of Last Node Death time for different initial energy values

From the above graphs it can be inferred that the initial energy of nodes when set as 5 Joule, it gives best results for the improved protocol. 


\section{Result Analysis}

The above simulations can be analyzed to infer following conclusions:

For different number of cluster-heads, the average increases in results of improved LEACH as compared to LEACH, LEACH-C and ALEACH are as follows:

Table 4 Result Analysis For Different Number Of Cluster-Heads

\begin{tabular}{|l|c|c|c|}
\hline \multirow{2}{*}{ Metrics } & \multicolumn{3}{|l|}{$\begin{array}{l}\text { Average Performance Increase of IMPROVED } \\
\text { LEACH as compared to }\end{array}$} \\
\cline { 2 - 4 } & LEACH & LEACH-C & ALEACH \\
\hline First Node Death time & $57 \%$ & $517 \%$ & $259 \%$ \\
\hline Half Node Death time & $42 \%$ & $83 \%$ & $105 \%$ \\
\hline Last Node Death time & $49 \%$ & $20 \%$ & $78 \%$ \\
\hline
\end{tabular}

For different amounts of initial energy, the average increase in results of improved LEACH as compared to LEACH is as follows:

Table 5 Result Analysis For Different Amounts Of Initial Energy
\begin{tabular}{|l|c|c|c|}
\hline \multirow{2}{*}{ Metrics } & \multicolumn{3}{|c}{$\begin{array}{l}\text { Average Performance Increase of } \\
\text { IMPROVED LEACH as compared to }\end{array}$} \\
\cline { 2 - 4 } & LEACH & LEACH-C & ALEACH \\
\hline First Node Death & $43 \%$ & $560 \%$ & $75 \%$ \\
\hline Half Node Death & $47 \%$ & $103 \%$ & $95 \%$ \\
\hline Last Node Death time & $50 \%$ & $43 \%$ & $67 \%$ \\
\hline
\end{tabular}

\section{Conclusion}

The main goal of this research revolves around design and implementation of an efficient routing protocol for WSNs. The existing LEACH protocol has been explored for the same and attempts to make it more energy-efficient have been made in this research and thesis. The improved protocol has been simulated under various simulation environments and the results collected have been compared with LEACH, LEACH-C and ALEACH protocols in the LEACH family. The research described in this thesis contributes towards development of an improved LEACH protocol which can lead to increase in the network life and make optimum use of the resources in the network.

\section{References}

[1]. Vidyasagar Potdar, Atif Sharif, Elizabeth Chang, "Wireless Sensor Networks: A Survey", International Conference on Advanced Information Networking and Applications Workshops, 2009, pp. 636-641.

[2]. Gaurav Sharma, Suman Bala, A. K. Verma, "Comparison of Flooding and Directed Diffusion for Wireless Sensor Network", India Conference (INDICON), Annual IEEE, 2009, pp. 1-4.

[3]. Yebin Chen Jian Shu Sheng Zhang Linlan Liu, "Data Fusion In Wireless Sensor Networks", Second International Symposium on Electronic Commerce and Security 2009, pp. 504-509.

[4]. C $\mathrm{Li}, \mathrm{M} \mathrm{Ye}, \mathrm{G}$ Chen, and J Wu, "An energy-efficient unequal clustering mechanism for wireless sensor networks," in IEEE International Conference on Mobile Ad hoc and Sensor Systems Conference, pp. 604, 2005.

[5]. C Y Wen and W A Sethares, "Automatic decentralized clustering for wireless sensor networks," EURASIP J.Wireless

[6]. Communication Network., vol. 5, no. 5, pp. 686-697, Oct.2005.

[7]. B. Krishnamachari, D. Estrin, and S. B. Wicker, "The impact of data aggregation in wireless sensor networks," in ICDCSW '02: $22^{\text {nd }}$ International Conference on Distributed Computing Systems. Washington, DC, USA:IEEE Computer Society, pp. 575-578, 2002.

[8]. Wendi Rabiner Heinzelman, Anantha Chandrakasan, and Hari Balakrishnan, "Energy-Efficient Communication Protocol for Wireless Microsensor Networks", Proceedings of the 33rd Hawaii International Conference on System Sciences (HICSS), Maui, HI, Jan. 2000, pp 1-10.

[9]. S. Lindsey and C. Raghavendra, "PEGASIS: Power-Efficient Gathering in Sensor Information Systems," IEEE Aerospace Conf. Proc., vol. 3, 9-16, 2002, pp. 1125-30.

[10]. Manjeshwar and D. P. Agarwal, “TEEN: a Routing Protocol for Enhanced Efficiency in Wireless Sensor Networks," 1st Int'l. Wksp. on Parallel and Distrib. Comp. Issues in Wireless Networks and Mobile Comp., April 2001, pp. 2009-2015.

[11]. Manjeshwar and D. P. Agarwal, "APTEEN: A Hybrid Protocol for Efficient Routing and Comprehensive Information Retrieval in Wireless Sensor Networks," Proc. Int'l. Parallel and Distrib. Proc. Symp., 2002, pp. 195-202.

[12]. O. Younis and S. Fahmy, "HEED: A Hybrid, Energy-Efficient, Distributed clustering approach for Ad Hoc sensor networks," IEEE Transactions on Mobile Computing, Vol. 3, No. 4, 2004, pp. 366-379.

[13]. Wendi B. Heinzelman, Anantha P. Chandrakasan and Hari Balakrishnan, "An Application-Specific Protocol Architecture for Wireless Microsensor Networks", 660 IEEE Transactions on Wireless Communicaitons, vol. 1, no. 4, October 2002, pp. 660-670.

[14]. Solaiman Ali Md, Dey Tanay, Biswas Rahul, "ALEACH: Advanced LEACH routing protocol for wireless microsensor networks", in Proceedings ICECE 2008, vols. 1 and 2, 2008, pp. 909-914.

[15]. Aimin Wang, Dailiang Yang, Dayang Sun, "A clustering algorithm based on energy information and cluster heads expectation for wireless sensor networks", Computers \& Electrical Engineering, Volume 38, Issue 3, May 2012, pp. 662-671. 